The University of San Francisco

USF Scholarship: a digital repository @ Gleeson Library | Geschke Center

Nursing and Health Professions Faculty Research and Publications

School of Nursing and Health Professions

$1-1-2015$

\title{
Auditory Hallucinations Interview Guide: Promoting Recovery with an Interactive Assessment Tool
}

Louise Nigh Trygstad

University of San Francisco, louisetrygstad@hotmail.com

Robin Kay Buccheri

University of San Francisco, buccherir@usfca.edu

Martha D. Buffum

San Francisco Veterans Affairs Medical Center, martibuffum@gmail.com

Dau-shen Ju

Iowa City Veterans Affairs Health Care System, daushen.ju@va.gov

Glenna A. Dowling

University of California, San Francisco, Glenna.Dowling@ucsf.edu

Follow this and additional works at: http://repository.usfca.edu/nursing_fac

Part of the Nursing Commons

\section{Recommended Citation}

Louise Nigh Trygstad, Robin Kay Buccheri, Martha D. Buffum, Dau-shen Ju, and Glenna A. Dowling. "Auditory Hallucinations Interview Guide: Promoting Recovery with an Interactive Assessment Tool" Journal of Psychosocial Nursing \& Mental Health Services 53.1 (2015).

This Article is brought to you for free and open access by the School of Nursing and Health Professions at USF Scholarship: a digital repository @ Gleeson Library | Geschke Center. It has been accepted for inclusion in Nursing and Health Professions Faculty Research and Publications by an authorized administrator of USF Scholarship: a digital repository @ Gleeson Library | Geschke Center. For more information, please contact repository@usfca.edu. 


\section{Auditory Hallucinations Interview Guide:}

\section{Promoting Recovery with an Interactive Assessment Tool}

Assessing patients who hear auditory hallucinations (AH) can be challenging. Psychiatricmental health nurses typically ask patients with known or suspected AH two questions: (a) Do you hear voices? and (b) Are your voices commanding you to harm yourself or anyone else? While these are critically important questions to help maintain the safety of the patient and the safety of others, there are other questions that should also be asked to obtain a comprehensive assessment of the voice hearer's experience. Such a thorough assessment of AH can help psychiatric-mental health nurses begin to understand what hearing voices is like for each patient, what strategies are effective for them in managing their $\mathrm{AH}$ and what help they may need from the staff.

To date, there has been no published interview guide with the following twofold purpose: 1) to help psychiatric-mental health professionals assess and plan care for each individual's voice hearing experience, and 2) to help patients tell their unique story of hearing voices. This paper describes the development and use of the Auditory Hallucinations Interview Guide (AHIG), a clinically useful assessment tool for the psychiatric-mental health nurse to collect assessment data that can be used to develop individualized care plans for voice hearers, including plans to ensure the safety of all. The AHIG helps voice hearers describe their experience of hearing voices from initial onset to current experiences including what makes their voices better and worse. The AHIG also teaches voice hearers and psychiatric-mental health nurses a shared language that increases their comfort level and facilitates effective communication when talking to each other about $\mathrm{AH}$. 


\section{BACKGROUND}

\section{Review of Existing Instruments to Assess AH}

Past methods to gain understanding about auditory hallucinations have included interactive semi-structured interviews, global measures of schizophrenia with subscales for auditory hallucinations, dimensions and characteristics of auditory hallucinations, and self-report measures. These methods are presented in two reviews of AH assessment tools (Frederick \& Killeen, 1998; Ratcliff, Farhall \& Shawyer, 2011).

The Frederick and Killeen (1998) review explored ten instruments developed between 1973 and 1995 to assess AH. Seven were clinician-rated and three involved self-report; nine were quantitatively measured while one was composed of open-ended questions about characteristics

of AH. What emerged from this review was the recognition of patients' capability to think about and discuss their symptoms and begin to understand the dimensions of auditory hallucinations. From the reviewers' perspective, two weaknesses included small sample sizes and the only studies were by the tools' authors. The reviewers reported that future research should include: larger samples for improved psychometric testing, inclusion of patient beliefs and attitudes, and inclusion of physiologic imaging studies and genetic dimensions.

The Ratcliff et al. (2011) review explored ten instruments developed to assess AH between 1998 and 2009. Two were structured interviews and eight were self-report. The two interviews overlap somewhat in quantitative measurement of AH characteristics. Differences involve measures of impact on self-esteem, compliance with commanding voices (without measuring presence of commanding voices), attention to voice content and perception of reality of voices. The authors concluded that most tools need refinement and more reliability and validity testing; more focus is needed on dimensions of clinical and theoretical concern including impact of the 
voices on distress, behavioral influence, and life disruption. Further, valuable information needs to be obtained from the voice hearers themselves.

In both sets of reviews the subjective experience of voice hearing is absent. Neither review discusses the interactive experience with any tool. While obtaining data for assessment, an actual interview as a clinical tool for establishing a therapeutic relationship is not elucidated in the literature.

\section{Development of AHIG}

In 1991, the second author spent a year as a visiting faculty member with the Symptom Management Faculty Group at the University of California, San Francisco. In 1992, the first two authors decided to focus their research using symptom management to help people with schizophrenia who have persistent AH learn how to manage their symptoms. There were few descriptions of the experience of $\mathrm{AH}$ found in the literature and most standardized psychiatric interviews (e.g., Structured Clinical Interview for DSM II-R- Patient Edition [SCID-P, Version 1]) asked very few questions about $\mathrm{AH}$.

The first part of the nursing process is always assessment and there were no evidence-based guidelines for nursing assessment of the experience of auditory hallucinations. In research language, this usually means a qualitative study is needed. Using all the descriptions of $\mathrm{AH}$ found in the literature, the dimensions of the experience (e.g., inside the head or outside the head; louder than my voice or quieter than my voice) were assembled to create an interview schedule to guide a qualitative study of the experience of $\mathrm{AH}$. This interview guide was used to answer the following research question: What is the experience of having persistent $\mathrm{AH}$ for people with schizophrenia? This was part of a larger pilot study that tested the 10-Session Behavior Management of Auditory Hallucinations Course (Buccheri, Trygstad, Kanas, Waldron \& 
Dowling, 1996).

\section{Theoretical Underpinnings of AHIG}

Peplau's theory of interpersonal relations. Comprehending the voice hearing experience requires genuine desire and interactive skill to learn the voice hearer's story. The interview method for understanding the AH experience is grounded in Peplau's interpersonal theory. Peplau's theory supports the use of communication skills that foster the therapeutic relationship between nurse and patient and specific roles of the nurse to create trust and foster growth (Peplau, 1989; Peplau, 1991). Using skills such as empathy, listening, and attentive and focused interest in a safe and comfortable environment, the nurse can conduct a comprehensive assessment with a person who hears AH. Using this interview guide, the person's story can be elicited and includes what name they give what they hear (e.g., voices, sounds, music), circumstances about when their voice(s) began, whether voices have continued and when, how many they hear, what makes them better or worse, whether the voice(s) are familiar, characteristics of the voices, amount of distress or pleasure, and, of particular importance, whether voices command them to harm themselves or others. Whether done all at one time or during multiple sessions, the assessment includes the identification of the person's needs relating to current symptoms (e.g., relief from distress) and begins a collaborative plan of care.

Some researchers purport that a voice hearer's understanding and meaning about their own voices are key to recovery (Place, Foxcroft \& Shaw 2011; Romme \& Escher, 2000). Describing experiences with voices to a caring clinician promotes comfort in feeling heard and a beginning understanding of oneself; these are among Peplau's critical elements for a therapeutic relationship (1991). The nurse helps clarify content to enable the patient to understand and 
interpret their own experience (Peplau, 1991). Nurses use these multiple interpersonal skills to assist patients in attaining their most optimal level of wellness.

Symptom management theory. The theory of symptom management was developed by the Symptom Management Faculty Group at the University of California, San Francisco (Humphreys et al., 2008). The purpose of the theory is to provide a framework for researchers and clinicians to collaborate in improving symptom assessment, symptom treatment and the outcome of treatment. The main concepts of symptom management theory are the symptom experience, symptom management strategies and symptom status outcomes. The theory describes the interaction of these three concepts and stresses the importance of examining these concepts within the context of the person (i.e., demographic, psychological, sociological, physiological, developmental), their environment (i.e., physical, social and cultural) and their health and illness (i.e., risk factors, health status, disease and injury).

These two theories provided a framework that guided the development of the AHIG. Peplau's theory of interpersonal relationships provided guidance for the relationship in which the AHIG should be administered — with respect for the person and their AH experience using nonjudgmental active listening to develop rapport and trust with the voice hearer. Administering a tool to assess $\mathrm{AH}$ in this manner helps develop the nurse-patient relationship so that the nurse and patient can then work together to assess the patient's experience of auditory hallucinations and find effective strategies to manage AH. Symptom management theory provided a comprehensive framework for the content of the AHIG. This theory guided the development of questions about the symptom experience, symptom management strategies and symptom status outcomes of using those strategies. The dimensions of the $\mathrm{AH}$ experience found in the literature 
together with these two theories were used to develop the AHIG--an interview guide to assess and learn about each individual's voice hearing experience.

\section{DESCRIPTION OF AHIG}

The AHIG is comprised of 31 opened-ended questions and one question with multiple yesno responses about the experience of hearing voices developed by the authors and reflecting the literature and their clinical experience. The AHIG is an assessment tool that can be used to create individualized interventions. It takes approximate $30-45$ minutes to complete. It is not a diagnostic tool nor does it result in a score. The AHIG can be used to complement other tools. The AHIG could be administered first to help develop rapport and trust then other assessment tools could be administered as needed.

The AHIG has been recently described in an article about the Self-Management of Unpleasant Voices-A Tested Practice Model. The AHIG is one of three assessment tools used in this model to assess the experience of auditory hallucinations (Buccheri et al., 2013) (See Table 1 for AHIG).

\section{Content of AHIG}

The experience of hearing voices is an internal and highly personal experience that is unique to each individual. The content of the AHIG provides important information about the dimensions of the experience for each individual. It establishes the presence of sounds (voices music and/or other) and whether it is believed others can hear these and whether the experience is distressing, positive, pleasurable or all of these. What each voice hearer has learned to do to cope, to make self more comfortable is explored. It covers the age of onset of hearing voices, what was happening in their life when the voices began, their current experience including the identity or anonymity of the voices, what the voices say, whether hostile or friendly or both, and 
commands from the voices including to harm self and/or others. Other questions about the voices include how long they talk, whether they talk to the hearer or about the hearer, the location of the voices, their loudness, frequency and clarity, time of day, place and situations where voices are usually better and worse, and the effects of medications, alcohol and drugs. History includes whether the voices have ever gotten much better or much worse and if so, what was the situation (or difference) at that time.

\section{Process of AHIG}

Building trust encourages the voice hearers to feel safe which is a primary goal of the interview. At the beginning of the interview, our script provides the following directions to promote trust and feelings of safety:

"From this interview I hope to better understand your experience of auditory hallucinations or hearing 'voices'. We have found from other patients' experiences and from what we have read in the literature that the experience of hearing voices can be quite varied and what helps can be quite varied. I hope that focusing on your experience might be helpful to you - that you might become aware of something about your experience that is useful to you and/or that sharing your experience might be helpful to you. If I ask any questions you do not want to answer, just say so - it is OK not to answer. And we will stop if you want to stop."

\section{FINDINGS}

\section{Findings from Two Decades: Reports and Observations from Using the AHIG}

The AHIG has been used for over 20 years in studies that evaluated outcomes of the 10Session Behavioral Management of Persistent Auditory Hallucinations Course. This 10-session course teaches voice hearers strategies to manage their auditory hallucinations (Buccheri et al., 
1996; Trygstad et al., 2002; Buccheri, Trygstad, \& Dowling, 2007). This evidence-based course has been widely disseminated in many countries (Buccheri \& Trygstad, 2012), across the USA and multiple times in Veterans Affairs facilities (Buffum et al. 2009; Buffum, Buccheri, Trygstad \& Dowling, 2014).

The clinicians who have participated in research evaluating this 10-session course used the AHIG and reported the voice hearer as more comfortable joining the course when the AHIG had been conducted as an introductory interview. Further, clinicians reported evidence of a bond between the interviewer and the voice hearer, thereby facilitating comfort during the course.

Some mental health clinicians who have used the interview guide have incorporated at least some of the questions into the admission interview form where they work. It is essential for staff to be aware of harm voices; it is useful to patients to have the experience of being understood and it is useful for patients and staff to have a common language and awareness.

One of the earliest observations in using the interview guide was the connection (rapport) established between the interviewer and the patient. This rapport still exists for the authors with patients who were interviewed in 1994 and 1995 and also those interviewed since then. Because of the knowledge the interviewer has of the patient from doing the AHIG and the shared experience in the 10-session course, it is easy for the psychiatric-mental health nurse to inquire about the well-being of the $\mathrm{AH}$ hearer and give support and suggestions based on knowing very specific information about each person.

In early interviews, patients repeatedly said they had never talked about their voices with anyone, many did not know that others heard voices. The individual interviews were consistently their first experience of someone being interested in having the AH hearers describe their experience in detail. Many AH hearers said they had never described the voices in this 
detail to themselves or anyone else. And although the interviewer always said they could decline to answer any question, AH hearers almost never did. They eagerly went on to the next question, curious what else there was to know.

A second useful observation was that almost all of the AH hearers liked to talk about their first experience with hearing voices. Onset of hearing $\mathrm{AH}$ almost always occurred during a stressful time of life (e.g., final exams, the invasion of Normandy, excessive drug use, divorce). The initial experience of hearing voices was almost always overwhelming and frightening. The voice hearers liked to tell this story of AH beginning over and over, both individually and in the 10-session course and it seemed to be part of their trying to understand their experience. And in the course, they liked to hear stories of how voices began for others. Although participants in the course had very different experiences regarding the onset of their voices, they almost always reported benefit from sharing their experiences.

A third useful observation was the high percentage of patients who heard voices to harm self or others. This was not part of the specific original research question and thus the data were not analyzed in the initial analysis. When this secondary analysis was completed, the researchers were surprised to find that almost half $(47 \%)$ of the voice hearers heard voices suggesting that they do harm: to themselves, to others or to both. This finding precipitated the development of the Harm Command Safety Protocol--a screening tool for assessing harm/safety when the psychiatric-mental health nurse learns that the patient hears harm voices that are commanding harm and they intend to act on those voices (Gerlock, Buccheri, Buffum, Trygstad, \& Dowling, 2010)

Findings from Using the AHIG: Demographics, Commonalities and Variations in Voice Hearing Experience 
The following is a summary of specific findings from a study that used the AHIG as a data collection tool with 62 participants (Trygstad et al., 2002).

\section{Demographics}

The ages of 10-session course participants ranged from 28 to 77 with a mean age of 46.05 (SD 11.65) years. Participants were multiethnic: $48 \%(n=10)$ White; 38\% $(n=8)$ Black or African American; 5\% (n=1) Chinese; 5\% (n=1) Filipino; and 5\% (n=1) Other. The majority of participants were single $(76 \%, n=16), 14 \%(n=3)$ were married and $10 \%(n=2)$ were divorced. None were employed full time but $19 \%$ did volunteer work.

The age when AH first started ranged from 1 to 37 years of age with a mean of 25.52 (SD 9.50) years. Some voice hearers cannot remember a time when they did not hear voices. They reported that they have heard voices for as long as they can remember. Others $(38 \%, \mathrm{n}=8)$ did not hear voices until they were at least 30 years old.

\section{Commonalities}

There were commonalities among voice hearers: the majority of voice hearers reported that AH were distressing to them. These unpleasant voices were usually derogatory and often suggested doing something harmful to themselves or others. Another commonality was the stressfulness of the onset and a felt need to keep their voice hearing a secret. Many patients talked about the stigma and isolation they experienced when their friends and family found out they heard voices. Voice hearers often report that they think they are the only ones who hear voices.

Many participants reported enjoying some of the voices (e.g., "they keep me company", "they laugh at my jokes", "they remind me to take a shower"). In teaching strategies to manage 
distressing voices, it has always been emphasized that voice hearers are welcome to keep any pleasant voices they want to keep; the purpose of teaching them behavioral strategies was the management of unpleasant or distressing voices.

Many participants reported that being in a structured situation like the 10-session course was helpful. For those who had had some of their AH go away, the consistent description was of gradual fading rather than abrupt cessation.

\section{Variations}

The number of voices heard varied from 1 to hundreds and from "occasionally" to " 24 hours a day 7 days a week". These descriptions remain accurate in our current work with people who hear AH. Variations in the AH experience also included the location of the voices, what was heard, the frequency of the voices, the identity of the voices, gender of the voices, whether they personally knew the voices and whether they were attributed to people that were dead or alive. Some voice hearers thought that the voices were coming from where they lived, only to learn after moving that their voices moved with them.

Differences were also noted in the time of day when voices were worst (e.g., on awakening, afternoon, evening and night), and whether voices awakened them from sleep or not. Effects of situations of low structure and low stimulation (e.g., being home alone) and very high stimulation (e.g., crowded bus or grocery store) also were reported differently but all of these usually made voices worse.

\section{What clinicians have learned from using AHIG}

Beneficial as an assessment tool, the AHIG can be used to guide interventions. When clinicians gather information from voice hearers, questions may trigger insight about their 
experiences that might not be readily apparent. For example, one AHIG question pertains to a time when voices stopped. Some voice hearers who were United States military veterans shared that their voices discontinued during the basic training and restarted afterwards. This change was attributed to the intensive physical and mental engagement and highly structured schedules. A number of similar questions in the AHIG explore the change and characteristics of $\mathrm{AH}$ throughout the day and across the life span. These questions bring to light that AH symptoms are not categorically static as voice hearers often report. The intensity, loudness, frequency, or tone of their voices, for example, could fluctuate depending on the time of the day, level of life engagement, presence of triggers, and general level of stress.

Voice hearers are often surprised by the AHIG interviews. They commonly report that they rarely had clinicians who explored their AH symptoms in such detail in the past. Some voice hearers paused and contemplated during the interview as if they never thought of their voices in such manner. Instead of responding to voices reactively and automatically as they often do, voice hearers begin a process of discovery about what the voices are and how they experience their voices. The self-awareness and insight they achieved during the AHIG interview paved the way for future intervention. For example, if an engaged life (i.e., basic training in the military) stopped their voices, what can a voice hearer do to increase life engagement that could potentially help to modulate their symptoms? If voices mimic the words a voice hearer's abusive father said to him, what choices would he have in response to the voices? Because of its role in self-discovery, the AHIG can be most beneficial if it is used at the early phase of a clinical relationship. It allows the voice hearers to be experts and to be engaged in a process of guided discovery with their clinicians.

The AHIG offers the opportunity for the clinician to listen, not to make assumptions. The 
following interview summary demonstrates how a clinician uses the AHIG to create a climate of trust by asking this client about his voices and listening to him tell his story while helping him understand the meaning of his experience.

Q: Do you remember our first visit? What do you remember about it?

A: You are the first person I told about my voices. Up until then, I only spoke briefly about them. I told you the bad time I had in a great deal of detail.

Q: What made you decide to share your experiences with me?

A: You made me very comfortable about talking with you. I thought I killed and raped my family when I first started having symptoms. When you have something for 6, 7 years, the relief you had about speaking with someone... It was a realistic fear. Every night you went to bed, you feared that you would wake up in hell. When I spoke with others, they looked at my alcohol history and thought my voices had to do with my drinking. I was already sober for a year! It was not because of my drinking. Some thought I had multiple personalities. They did not listen.

Q: Were there questions you remember the most about that visit?

A: Spirituality. It was never brought up before. For me, my struggle was about heaven and hell, about morality. Like the Babylonian King [Nebuchadnezzar] who became insane and saved by God, mine is a spirituality journey.

\section{Implications for Nursing Practice}

There are many benefits of using the AHIG. These benefits include allowing voice hearers to share their experience of $\mathrm{AH}$ in a safe, structured manner. The AHIG provides structure to psychiatric-mental health nurses to invite patients to answer detailed questions during an individual interview about the onset of their voices and the voice hearing experience. These 
questions include asking about triggers to $\mathrm{AH}$ and strategies they use to help manage their $\mathrm{AH}$ including commands to harm self and others.

Using the AHIG allows the psychiatric-mental health nurse the opportunity to convey empathy and employ good listening skills that helps to build trust between the voice hearer and the mental health professional. The AHIG provides shared terminology for experiences that may have been difficult for the voice hearer to describe to others. This terminology can then be shared with other mental health professionals working with the voice hearer toward recovery. For example, information collected with the AHIG can be used to develop an individualized care plan for the voice hearer. This care plan could include that the voice hearer prefers to have his voices called "thoughts" or that he hears commands to harm self but has never acted on them.

Students in psychiatric nursing often have a difficult time trying to talk to persons with schizophrenia - it certainly is not like talking with one of their friends. The structure of the AHIG allows students and patients to feel more comfortable talking to one another. Students are often fascinated with what they learn during the interview and pleased to be able to connect with patients; patients feel heard, and soon the interaction progresses easily within the structure.

\section{Implications for Nursing Research}

All tools require ongoing evaluation. We continuously seek feedback from clinicians who have incorporated the AHIG into their work with voice hearers in inpatient and outpatient settings. Any changes for brevity, flow, and content can then be re-evaluated; clinicians often 
make alterations that better fit their situations. Hence, we welcome others to use the AHIG after reading this article and to submit feedback to us (See Table 2 for Feedback Form).

Future research is needed for establishing usefulness of the AHIG for mental health clinicians from all disciplines in inpatient and community residential care settings, informal caregivers (e.g., support groups), and family members. Additionally, continuity of care evaluation is needed about utility of the individualized care plan developed from the AHIG information.

\section{CONCLUSIONS}

Psychiatric-mental health nurses need to learn more about each voice hearer's past and current experiences so they can provide individualized nursing care. The AHIG provides a structured 30-45 minute interview that can be used to assess the voice hearer's past and current symptoms and to develop a therapeutic relationship. The AHIG contributes a theory-based, clinically tested, structured interview guide to the psychiatric-mental health nurses' repertoire of assessment and communication tools. The tool has benefits for staff, nursing students and voice hearers. Utilizing the valuable communication skills of listening and conveying empathy, the psychiatric-mental health nurse can establish trust and truly enable the patient's comfort for being open and honest about hearing their voices.

\section{References}

Buccheri, R., \& Trygstad (2012). Evidence-based psychiatric nursing for community-based patients-behavioral management of auditory hallucinations [published keynote in Japanese: $24^{\text {th }}$ Annual Japan Academy of Psychiatric and Mental Health Nursing Conference, 
Kumamoto, Japan, June 23 $\left.{ }^{\text {rd }}, 2012\right]$. The Journal of Mental Health and Psychiatric Nursing, 21(2), 52-71.

Buccheri, R. K., Trygstad, L. N., Buffum, M. D., Birmingham, P., \& Dowling, G. A. (2013). Self-management of unpleasant auditory hallucinations: A tested practice model. Journal of Psychosocial Nursing and Mental Health Services, 51(11), 26-34.

Buccheri, R. K., Trygstad, L., Kanas, N., Waldron, B., Dowling G. (1996). Auditory hallucinations in schizophrenia: Group experience in examining symptom management and behavioral strategies. Journal of Psychosocial Nursing and Mental Health Services, 34, 1225.

Buccheri, R., Trygstad, L., \& Dowling, G. (2007). Behavioral management of command hallucinations to harm in schizophrenia. Journal of Psychosocial Nursing and Mental Health Services, 45(9), 46-54.

Buffum, M. D., Buccheri, R. K., Trygstad, L. N. \& Dowling, G. A. (2014). Disseminating evidence-based care of auditory hallucinations. Journal of Psychosocial Nursing and Mental Health Services, 52(4), 32-41.

Buffum, M. D., Buccheri, R., Trygstad, L., Gerlock, A. A, Birmingham, P., Dowling, G. A., \& Kuhlman, G. J. (2009). Behavioral management of auditory hallucinations: Implementation and evaluation of a 10-week course. Journal of Psychosocial Nursing and Mental Health Services, 47(9), 32-40.

Frederick, J.A. \& Killeen, M.R. (1998). Instruments for assessment of auditory hallucinations. Archives of Psychiatric Nursing, 12(5), 255-263. 
Humphreys, J., Lee, K.A., Carrieri-Kohlman, V., Puntillo, K., Facucett, J., Janson, S.,...the UCSF School of Nursing Symptom Management Faculty Group. (2008). Theory of symptom management. In M. J. Smith \& P. R. Liehr (Eds.), Middle range theory for nursing ( $2^{\text {nd }}$ ed.) (pp. 145-158). New York: Springer Publishing.

Peplau, H.E. (1989). Future directions in psychiatric nursing from the perspective of history. Journal of Psychosocial Nursing and Mental Health Services, 27(2), 18-21, 25-8.

Peplau, H.E. (1991). Interpersonal relations in nursing: A conceptual frame of reference for psychodynamic nursing. New York: Springer.

Place, C., Foxcroft, R., \& Shaw, J. (2011). Telling stories and hearing voices: Narrative work with voice hearers in acute care. Journal of Psychiatric and Mental Health Nursing, 18, 837-842.

Ratcliff, K., Farhall, J., \& Shawyer, F. (2011). Auditory hallucinations: A review of assessment tools. Clinical Psychology and Psychotherapy, 18, 524-534.

Romme, M., \& Escher, S. (2000). Making sense of voices. Mind: London.

Trygstad L., Buccheri, R., Dowling, G., Zind, R., White, K., Griffin, J., et al. (2002). Behavioral management of persistent auditory hallucinations in schizophrenia: Outcomes from a 10week course. Journal of the American Psychiatric Nurses Association, 8(3), 84-91. 